\title{
A FRACTIONALLY INTEGRATED EXPONENTIAL MODEL FOR U.K. UNEMPLOYMENT
}

\author{
L.A. GIL-ALANA
}

Institut für Statistik und Ökonometrie, Humbold Universität zu Berlin, Berlin, Germany. University of Navarre, Department of Economics, Pamplona, Spain.

\begin{abstract}
Fractionally integrated models with the disturbances following a Bloomfield (1973) exponential spectral model are proposed in this article for modelling the U.K. unemployment. This enables us a better understanding of the low-frequency dynamics affecting the series, without relying on any particular ARMA specification for its short-run components, which, in general, requires many more parameters to estimate. The results indicate that this exponential model, confounded with fractional integration, may be a feasible way of modelling unemployment, also showing that its order of integration is much higher than one and thus, leading to the conclusion that the standard practice of taking first differences may lead to erroneous results.
\end{abstract}

Key words: Fractional integration; Long memory; Unemployment JEL Classification: C22 


\section{INTRODUCTION}

In this article, an alternative way of modelling the U.K. unemployment by means of combining fractional integration with the Bloomfield (1973) exponential spectral model for the disturbances is proposed. The main motivation for using the fractional integration framework is that it seems much more general than the traditional approaches based on deterministic I(0) or unit roots I(1) models. We use the exponential spectral model of Bloomfield (1973) because the usual ARMA representations require many more parameters to estimate compared with this non-parametric approach, which also produces autocorrelations decaying exponentially as in the AR processes.

The article is organised as follows: Section 2 briefly describes the concepts of fractional integration and of the exponential spectral model of Bloomfield (1973). Section 3 shows the method of estimating the parameters in this context by using the Whittle function, (which is an approximation to the likelihood function), and we also present a version of the tests of Robinson (1994) for testing these type of models. In Section 4 we estimate and test the models using different measures of the U.K. unemployment while Section 5 contains some concluding remarks.

\section{AN EXPONENTIAL SPECTRAL FRACTIONALLY INTEGRATED MODEL}

Many economic time series contain plenty of evidence of nonstationarity and much controversy in macroeconomics has revolved around the question of the suitability of I(1) or unit root models for describing raw time series as opposed to the so-called trendstationary models, where the raw series is described as an $\mathrm{I}(0)$ process plus a deterministic trend. Unit roots and linear time trends each constitute extremely specialised models for nonstationarity, but each has the advantage of conceptual and computational simplicity, and they are naturally thought of as rival models because a unit root, with or without a drift, 
implies a constant or linear trend function, the distinction then being in the disturbance terms.

However, in the last few years, an increasing amount of literature studying the source of nonstationarity in macroeconomic time series in terms of fractionally differenced time series, has appeared. We can consider a process like

$$
(1-L)^{d} x_{t}=v_{t}, \quad t=1,2, \ldots
$$

where $v_{t}$ is an $I(0)$ covariance stationary process with spectral density which is positive and finite at zero frequency. Clearly, if $\mathrm{d}=0, \mathrm{x}_{\mathrm{t}}=\mathrm{v}_{\mathrm{t}}$ and the process is "weakly dependent" as opposed to the "strong dependence" case when $d>0$. The macroeconomic literature stresses the cases $d=0$ and $d=1$ but we can define (1) for any real $d$ by the expansion

$$
(1-L)^{d}=1+\sum_{j=1}^{\infty} \frac{\Gamma(d+1)(-L)^{j}}{\Gamma(d-j+1) \Gamma(j+1)} .
$$

If $0<\mathrm{d}<0.5$ in (1) then, $\mathrm{x}_{\mathrm{t}}$ is a covariance stationary process, having autocovariances which decay much more slowly than those of an ARMA process, in fact so slowly as to be nonsummable. Models such as (1) provide a type of flexibility in modelling low-frequency dynamics not achieved by non-fractional ARIMA models, and stationary fractional models have been shown by Granger (1980), Robinson (1978), to arise from aggregation of ARMA series with randomly varying coefficients.

On the other hand, AR modelling of the $\mathrm{I}(0)$ process $\mathrm{v}_{\mathrm{t}}$ is very conventional, but many other types of $\mathrm{I}(0)$ process exist, including ones outside the stationary and invertible ARMA case. We propose in this paper the use of the exponential spectral model of Bloomfield (1973), in which $\mathrm{v}_{\mathrm{t}}$ is defined exclusively in terms of its spectral density function, given by

$$
f(\lambda ; \tau)=\frac{\sigma^{2}}{2 \pi} \exp \left(2 \sum_{r=1}^{m} \tau_{r} \cos (\lambda r)\right)
$$


Suppose that $\mathrm{v}_{\mathrm{t}}$ follows an ARMA process of form

$$
v_{t}=\sum_{r=1}^{p} \phi_{r} v_{t-r}+\varepsilon_{t}-\sum_{r=1}^{q} \theta_{r} \varepsilon_{t-r}
$$

where $\varepsilon_{\mathrm{t}}$ is a white noise process and all zeros of $\phi(\mathrm{L})$ lying outside the unit circle and all zeros of $\theta(\mathrm{L})$ lying outside or on the unit circle. Clearly, the spectral density function of this process is then

$$
f(\lambda ; \varphi)=\frac{\sigma^{2}}{2 \pi}\left|\frac{1-\sum_{r=1}^{q} \theta_{r} e^{i r \lambda}}{1-\sum_{r=1}^{p} \phi_{r} e^{i r \lambda}}\right|^{2},
$$

where $\varphi$ corresponds to all the AR and MA coefficients and $\sigma^{2}$ is the variance of $\varepsilon_{t}$. Bloomfield (1973) showed that the logarithm of an estimated spectral density function is often found to be a fairly well-behaved function and can thus be approximated by a truncated Fourier series. He showed that (2) approximates (3) well where p and q are of small values, which usually happens in economics. Like the stationary AR(p) model, this has exponentially decaying autocorrelations and thus, using this specification, we do not need to rely on so many parameters as in the ARMA processes, which always results tedious in terms of estimation, testing and model specification.

The Bloomfield model for I(0) processes, confounded with the fractional model (1) has not been used very much in previous econometric applications (though the Bloomfield model itself is a well-known model in other disciplines, eg. Beran, 1993), and one byproduct of this work is its emergence as a credible alternative to the fractional ARIMAs which have become conventional in parametric modelling of long memory. Among the few examples found in the literature are Gil-Alana and Robinson (1997) and Velasco and Robinson (1999). The following section shows the method of estimating and testing the 
parameters in this context of fractionally integrated models with Bloomfield (1973) exponential spectral disturbances.

\section{ESTIMATION AND TESTING IN THE FREQUENCY DOMAIN}

Given a covariance stationary process $\left\{\mathrm{x}_{\mathrm{t}}, \mathrm{t}=1,2, \ldots\right\}$, where $\mathrm{x}_{\mathrm{t}}$ is given by (1) (with $\mathrm{d}<$ $0.5)$ and $v_{t}$ is an $I(0)$ process with spectral density function of form as in (2), we are firstly concerned with the estimation of the parameters of the model, that is, $d$ and those appearing in (2). Based on parametric approaches, $d$ is estimated jointly with all the other parameters that specify the model. Since $v_{t}$ is defined in terms of its spectral density function, the estimation must be carried out in the frequency domain.

Fox and Taqqu (1986) assumed Gaussianity, and minimized the Whittle function (an approximation to the exact likelihood function) of a covariance stationary process with $\mathrm{I}(0)$ disturbances of a very general form (and thus, including the Bloomfield (1973) exponential spectral model). Calling $\psi$ the parameter vector to be estimated, they minimized

$$
\frac{1}{4 \pi} \int_{-\pi}^{\pi}\left(\log f(\lambda ; \psi)+\frac{I(\lambda)}{f(\lambda ; \psi)}\right) d \lambda
$$

where $I(\lambda)$ is the periodogram of the process $x_{t}$, defined as

$$
I(\lambda)=\frac{1}{2 \pi T}\left|\sum_{t=1}^{T} x_{t} e^{i \lambda t}\right|^{2}
$$

The estimate was shown to be consistent and asymptotically normal under appropriate conditions, which are satisfied by the fractional model (1) with $0<\mathrm{d}<0.5$. Furthermore, Velasco and Robinson (1999) show a way of estimating d for nonstationary series with 0.5 $\leq \mathrm{d}<1$, and even for any degree of nonstationary $(\mathrm{d} \geq 0.5)$ by means of tapering. Another 
estimate with the same asymptotic behaviour is obtained if (4) is replaced by a sum over the Fourier frequencies, i.e. minimizing

$$
\frac{1}{2 T} \sum_{j=1}^{T-1}\left(\log f\left(\lambda_{j} ; \psi\right)+\frac{I\left(\lambda_{j}\right)}{f\left(\lambda_{j} ; \psi\right)}\right)
$$

with $\lambda_{\mathrm{j}}=2 \pi \mathrm{j} / \mathrm{T}$. In the model given by (1) and (2), $\psi=\left(\mathrm{d}, \tau^{\prime}\right)^{\prime}$, and

$$
f\left(\lambda_{j} ; \psi\right)=\frac{\sigma^{2}}{2 \pi} \frac{1}{\left|1-e^{i \lambda_{j}}\right|^{2 d}} \exp \left(2 \sum_{r=1}^{m} \tau_{r} \cos \left(\lambda_{j} r\right)\right)
$$

Substituting now (6) in (5), the minimum in (5) can be easily carried out through a computer programme.

We next describe a testing procedure suggested by Robinson (1994) to test the order of integration in raw time series in this context of exponential spectral disturbances. Suppose we observe $\left\{\mathrm{y}_{\mathrm{t}}, \mathrm{t}=1,2, \ldots \mathrm{T}\right\}$, where

$$
y_{t}=\beta^{\prime} z_{t}+x_{t}, \quad t=1,2, \ldots \ldots, T
$$

and $z_{t}$ is a $(k x 1)$ vector of exogenous regressors, (like $z_{t}=(1, t)$ ' to include, for instance, the case of a linear time trend); $x_{t}$ is described by (1) with the disturbances following a spectral density function as in (2). In general, we wish to test the null hypothesis

$$
H_{o}: d=d_{o}
$$

for a given real number $d_{0}$. When $z_{t}$ is nonempty, we form

$$
w_{t}=(1-L)^{d_{o}} z_{t},
$$

taking

$$
z_{t}=0, \quad t \leq 0
$$

Based on the null model, the least-squares estimate of $\beta$ and residuals are

$$
\hat{\beta}=\left(\sum_{t=1}^{T} w_{t} w_{t}{ }^{\prime}\right)^{-1} \sum_{t=1}^{T} w_{t}(1-L)^{d_{o}} y_{t},
$$




$$
\hat{v}_{t}=(1-L)^{d_{o}} y_{t}-\hat{\beta}^{\prime} w_{t}, \quad t=1,2, \ldots \ldots
$$

and its periodogram is

$$
P_{\hat{v}}(\lambda)=\frac{1}{2 \pi T}\left|\sum_{t=1}^{T} \hat{v}_{t} e^{i \lambda t}\right|^{2}
$$

Unless $\mathrm{f}$ in (2) is a completely known function, (e.g., as when $\mathrm{v}_{\mathrm{t}}$ is white noise), we have to estimate the nuisance parameter vector $\tau$. The estimate must be a Gaussian one, that is, it must have the same limit distribution as the efficient maximum likelihood estimate based on the assumption that $v_{1}, v_{2}, \ldots, v_{T}$, is Gaussian. One such estimate, which fits naturally into the frequency domain setting, is

$$
\hat{\tau}=\arg _{\tau} \min _{\tau} \sigma^{2}(\tau)
$$

where the minimization is carried out over a suitable subset of $\mathrm{R}^{\mathrm{m}}$, and

$$
\sigma^{2}(\tau)=\frac{2 \pi}{T} \sum_{j=1}^{T-1} P_{\hat{v}}\left(\lambda_{j}\right) / \exp \left(2 \sum_{r=1}^{m} \tau_{r} \cos \left(\lambda_{j} r\right)\right)
$$

Next we form

$$
m\left(\lambda_{j}\right)=\log \left|2 \sin \frac{\lambda_{j}}{2}\right|
$$

and the test statistic is

$$
\hat{s}=\frac{T}{\hat{b}} \frac{\hat{a}}{\hat{\sigma}^{2}},
$$

where

$$
\begin{gathered}
\hat{\sigma}^{2}=\sigma^{2}(\hat{\tau}) \\
\hat{a}=\frac{-2 \pi}{T} \sum_{j=1}^{T-1} m\left(\lambda_{j}\right) P_{\hat{v}}\left(\lambda_{j}\right) / \exp \left(2 \sum_{r=1}^{m} \tau_{r} \cos \left(\lambda_{j} r\right)\right)
\end{gathered}
$$

and 


$$
\hat{b}=\sum_{j=m+1}^{\infty} j^{-2}=\frac{\pi^{2}}{6}-\sum_{j=1}^{m} j^{-2}
$$

Under the null hypothesis (8), Robinson (1994) established under regularity conditions that

$$
\hat{s} \quad \rightarrow_{d} N(0,1) \quad \text { as } T \rightarrow \infty \text {. }
$$

The conditions on $v_{t}$ in (10) are far more general than Gaussianity, with a moment condition only of order 2 required. An approximate one-sided $100 \alpha \%$-level test of (8) against alternatives

$$
H_{1}: d>d_{o}
$$

is given by the rule:

$$
\text { "Reject } \mathrm{H}_{\mathrm{o}} \text { if } \hat{s}>z_{\alpha} \text { ", }
$$

where the probability that a standard normal variate exceeds $z_{\alpha}$ is $\alpha$. Conversely, an approximate one sided $100 \alpha \%$ level test of (8) against alternatives

$$
H_{1}: d<d_{o}
$$

is given by the rule:

$$
\text { "Reject } \mathrm{H}_{\mathrm{o}} \text { if } \hat{s}<-z_{\alpha} \text { ". }
$$

As these rules indicate, we are in a classical large sample testing situation for reasons described by Robinson (1994), who also showed that the above tests are efficient in the Pitman sense that against local alternatives $H_{1}: d=d_{o}+\delta T^{-1 / 2}$ for $\delta \neq 0$, the test has an asymptotic normal distribution with variance 1 and mean which cannot (when $\mathrm{v}_{\mathrm{t}}$ is Gaussian) be exceeded in absolute value by that of any rival regular statistic.

In the following section, a fractionally integrated Bloomfield (1973) model is estimated and tested using different measures of the U.K. unemployment. All calculations 
were carried out using Fortran. A diskette containing the codes for the estimation and testing programmes is available from the author on request.

\section{AN EMPIRICAL APPLICATION TO THE U.K. UNEMPLOYMENT}

Four different measures of unemployment were considered. Firstly, we looked at the number of people claiming unemployment benefits. This measure is known as the claimant count (CC) and is available monthly. We look at this series $\left(\mathrm{U}_{\mathrm{t}}\right)$ and also at its logarithmic transformation $\left(\log U_{t}\right)$. Another measure, which is related to the unemployment rate, is the CC series as a percentage of the workforce. We also look at this series, $\left(\mathrm{u}_{\mathrm{t}}\right)$, as well as its logistic transformation:

$$
u_{t}^{*}=\log \left(\frac{u_{t}}{1-u_{t}}\right) .
$$

All these monthly series start in January 1971 and end in August 1998. These series have been investigated in a number of papers by Gil-Alana (1999a,b,c), studying their orders of integration in terms of non-parametric and parametric (ARFIMA) models. This article is therefore a complementary work in that direction. In all these previous works, the order of integration of the U.K. unemployment was found to be much higher than one and thus, rejecting the hypothesis of a unit root.

In this article we want to investigate the order of integration of the series when the disturbances follow a Bloomfield (1973) exponential spectral model. Across Tables 1-4 we present the estimated values of $d$ when $x_{t}$ is given by (1) and $v_{t}$ follows the Bloomfield (1973) model (2) with $\mathrm{m}=0$ (i.e., $\mathrm{v}_{\mathrm{t}}$ is white noise); 1; 2 and 3. These estimates were found minimizing (5) using a grid search over the range $[-5,5]$ for $\tau$ and $[-0.5,0.5)$ for $d$ of length 0.01 with $\mathrm{x}_{\mathrm{t}}$ based on the second differences. In all these tables we also display the estimated values of $d$ when $v_{t}$ follows an $\operatorname{AR}(p)$ process with $p=0 ; 1 ; 2$ and 3 . The 
estimation in these cases was carried out using the Sowell's (1992) procedure of estimating by maximum likelihood in the time domain. Thus, the difference observed in the estimated values of $d$ when $p$ and $m$ are both equal to zero is clearly due to the different method of estimation used.

Starting with $U_{t}$, we observe in Table 1 that if $v_{t}$ is white noise, the estimated value of $\mathrm{d}$ is 1.66 when using the time domain estimation procedure, and 1.65 when using the frequency domain approach. Allowing $\mathrm{v}_{\mathrm{t}}$ to be weakly parametrically autocorrelated, the order of integration of $U_{t}$ seems to be higher, ranging between 1.83 and 2.05 when modelling $\mathrm{v}_{\mathrm{t}}$ with autoregressions but slightly greater, and ranging between 1.92 and 2.20 when $\mathrm{v}_{\mathrm{t}}$ follows the Bloomfield (1973) exponential spectral model. Thus, we observe that the order of integration of this series is much higher than one, fluctuating around 2 when the disturbances are weakly autocorrelated.

\section{(Tables 1 and 2 about here)}

Table 2 displays the results for $\log U_{t}$. Again all the values are higher than 1 . If $v_{t}$ is white noise, the estimated value of $\mathrm{d}$ is now 1.63 in the time domain and 1.62 in the frequency domain. If $\mathrm{v}_{\mathrm{t}}$ is an $\mathrm{AR}$ process, $\mathrm{d}$ oscillates between 1.71 and 1.84, and modelling $\mathrm{v}_{\mathrm{t}}$ with Bloomfield (1973), the values of $\mathrm{d}$ oscillate between 1.73 and 2.01. Thus, we again observe a higher value when using the exponential spectral model. Comparing these results with those in Table 1 we observe that using the logarithmic transformation, the orders of integration are slightly smaller though still greatly above one.

Taking $\mathrm{u}_{\mathrm{t}}$ as the measure of unemployment, the results are given in Table 3 . If $\mathrm{v}_{\mathrm{t}}$ is white noise, the estimated value of $d$ is in both cases around 1.50. That means that if we take first differences, the differenced series behaves as in the boundary case between stationarity and nonstationarity. Allowing $\mathrm{v}_{\mathrm{t}}$ to be weakly autocorrelated, the values range 
between 1.70 and 1.92 when using autoregressions, and between 1.87 and 2.08 with the exponential spectral disturbances.

\section{(Tables 3 and 4 about here)}

Finally, using the logistic transformation of $\mathrm{u}_{\mathrm{t}},\left(\mathrm{u}_{\mathrm{t}}{ }_{\mathrm{t}}\right)$ as the measure for unemployment, we again observe that if $\mathrm{v}_{\mathrm{t}}$ is white noise, the estimated value of $\mathrm{d}$ is around 1.50 but allowing weak dependence in the disturbances, the values of $d$ are slightly higher, ranging between 1.64 and 1.81 when $\mathrm{v}_{\mathrm{t}}$ is $\mathrm{AR}$ and between 1.69 and 2.01 with the Bloomfield (1973) model.

We can conclude the analysis of these four tables by saying that when estimating the order of integration of the U.K. unemployment, the value of $d$ seems to be much higher than one. Given that both methods of estimation are based on maximum likelihood, conventional tests based on the statistic $(\hat{d}-d) / S E(\hat{d})$ were performed, rejecting the unit root null (ie., $\mathrm{d}=1$ ) in all cases across all series. If the disturbances are white noise, the order of integration is slightly higher than 1.60 for $U_{t}$ and $\log U_{t}$, and is around 1.50 for $\mathrm{u}_{t}$ and $\mathrm{u}_{\mathrm{t}}^{*}$. If we allow the disturbances to be weakly parametrically autocorrelated, the orders of integration are even higher, fluctuating between 1.70 and 2.20 in all cases. We also observe higher values when using the Bloomfield (1973) exponential spectral model rather than the autoregressions, though the difference between them is in all cases smaller than 0.15 .

Across Tables 5-8 we test the order of integration of the series using the tests of Robinson (1994) described in Section 3. Denoting any of the measures of unemployment $\mathrm{y}_{\mathrm{t}}$, we employ throughout the model (1) and (7) with $\mathrm{z}_{\mathrm{t}}=(1, \mathrm{t})^{\prime}, \mathrm{t} \geq 1, \mathrm{z}_{\mathrm{t}}=(0,0)^{\prime}$ ' otherwise, SO

$$
\begin{array}{ll}
y_{t}=\alpha+\beta t+x_{t}, & t=1,2, \ldots \\
(1-L)^{d} x_{t}=v_{t}, & t=1,2, \ldots,
\end{array}
$$


treating separately the cases $\alpha=\beta=0$ a priori, (i.e., including no regressors in the undifferenced regression); $\alpha$ unknown and $\beta=0$ a priori, (i.e., including an intercept); and finally, $\alpha$ and $\beta$ unknown (i.e., including a linear time trend). We model the $\mathrm{I}(0)$ process $\mathrm{v}_{\mathrm{t}}$ to be both white noise $(\mathrm{m}=0)$ and weakly autocorrelated with the Bloomfield (1973) model of orders 1, 2 and 3. Clearly, if $v_{t}$ is white noise, when $d=1$, the differences $(1-L) y_{t}$ behave, for $\mathrm{t}>1$, like a random walk when $\beta=0$, and a random walk with a drift when $\beta \neq 0$. However, we report test statistics not merely for the null $d_{o}=1$ in (8) but for $d_{o}$ $=1.20 ; 1.40 ; 1.50 ; 1.60 ; 1.80$ and 2 .

The test statistic reported in Table 5 (and also in Tables 6-8) is the one-sided one given by (9), so that significantly positive values of this, see (12), are consistent with (11) whereas significantly negative ones, see (14), are consistent with (13). A notable feature observed across the tables is that $\hat{s}$ is in all the cases monotonically decreasing with respect to $d_{0}$. This is something that we should expect of any reasonable statistic, given correct specification and adequate sample size, because, for example, we would wish that if $d=$ 1.20 is rejected against $\mathrm{d}>1.20$, an even more significant result in this direction would be obtained when $\mathrm{d}=1$ is tested.

Starting with $U_{t}$ in Table 5, the first thing we observe is that the nulls $d=1$ and $d=$ 1.20 are both rejected in all cases in favour of alternatives with $d>1.20$. Also, $d=1.40$ is always rejected except for white noise $\mathrm{v}_{\mathrm{t}}$ and $\alpha=\beta=0$. If we do not include regressors and $v_{t}$ follows a Bloomfield (1973) model, the values of $d$ where $H_{o}(8)$ is not rejected are 1.50 and 1.60 when $\mathrm{m}=1 ; 1.50,1.60$ and 1.80 when $\mathrm{m}=2$, and all these values along with 2.00 when $\mathrm{m}=3$. If we include an intercept or a linear time trend in (7) the results seem more conclusive: $d=1.60$ is the only non-rejection case for white noise $v_{t} ; d=1.80$ and 2 are not rejected with Bloomfield (1973) disturbances and $\mathrm{m}=1$ and 2; and $\mathrm{d}=2$ is the only nonrejection case with $m=3$. These results are clearly consistent with those given in Table 1 , 
where the order of integration was found to be around 1.60 for white noise disturbances and ranging between 1.80 and 2 for the exponential spectral model of Bloomfield (1973).

\section{(Tables 5 and 6 about here)}

Table 6 reports the results for the $\log U_{t}$. The most striking point we observed here is that the null $\mathrm{d}=1$ is not rejected in any case when $\alpha=\beta=0$ a priori, however, including regressors, this hypothesis is strongly rejected in favour of alternatives with d equal to or greater than 1.50. This might relate to the fact that $(1-\mathrm{L})^{\mathrm{d}} 1$ tends to zero for all positive $\mathrm{d}$ smaller than 1 and this is faster as $d$ aproximates 1 , becoming exactly zero when $d=1$. Thus, it might be the case that when testing for a unit root, the model should include an intercept rather than imposing $\alpha=\beta=0$. We see that including an intercept, $\mathrm{H}_{\mathrm{o}}(8)$ is not rejected for $d=1.60$ with $\mathrm{m}=0$ and ranges between 1.50 and 2 with the Bloomfield (1973) disturbances.

\section{(Tables 7 and 8 about here)}

Tables 7 and 8 correspond respectively to $\mathrm{u}_{\mathrm{t}}$ and $\mathrm{u}_{\mathrm{t}}^{*}$. Starting with $\mathrm{u}_{\mathrm{t}}$, we again observe that the values of $d$ fluctuate between 1.40 and 2 . If $v_{t}$ is white noise, $d=1.50$ is the only case where $\mathrm{H}_{\mathrm{o}}$ is not rejected, which is completely in line with the estimation carried out in Table 3. Similarly, if $\mathrm{v}_{\mathrm{t}}$ is weakly autocorrelated and we include regressors, the nonrejection values are $\mathrm{d}=1.80$ when $\mathrm{m}=1 ; \mathrm{d}=2$ when $\mathrm{m}=2$; and $\mathrm{d}=1.60,1.80$ and 2 when $\mathrm{m}=3$, which is once more consistent with Table 3. Finally, measuring unemployment in terms of $\mathrm{u}_{\mathrm{t}}^{*}$, the results are very similar to those given in Table 6 (for $\log \mathrm{U}_{\mathrm{t}}$ ). If we do not include regressors, the unit root null hypothesis is not rejected along with other fractionally hypotheses with $d>1$. However, including an intercept or a linear time trend, all the nonrejections occur when $d \geq 1.50$, clearly showing the nonstationary character of the series.

We can conclude by saying that for all these measures of unemployment, the orders of integration seem higher than one, and this is observed whether or not we include 
deterministic regressors like an intercept and/or a linear time trend in the model. Thus, though we do not stress in this article any particular specialized model for any series, these results, as a whole, show that the standard practice of taking first differences when modelling the U.K. unemployment may still lead to series with a component of long memory behaviour.

\section{CONCLUSIONS}

A fractionally integrated model with the disturbances following a Bloomfield (1973) exponential spectral model has been proposed in this article for modelling the U.K. unemployment. This type of model can be considered as an alternative to the mostcommonly used fractionally ARIMA (ARFIMA) ones, with the Bloomfield (1973) structure describing the short-run dynamics without need of estimating so many parameters as in the ARMA case. A method based on the Whitle function for estimating by maximum likelihood in the frequency domain, along with a procedure suggested by Robinson (1994) for testing these type of models were performed using four different measures of unemployment. These measures were: the number of people claiming unemployment benefits, $\left(\mathrm{U}_{\mathrm{t}}\right)$; its logarithmic transformation, $\left(\log \mathrm{U}_{\mathrm{t}}\right)$; the number of people claiming benefits as a percentage of the workforce, $\left(\mathrm{u}_{\mathrm{t}}\right)$; and its logistic transformation, $\left(\mathrm{u}_{\mathrm{t}}{ }_{\mathrm{t}}\right)$.

Using an estimation procedure based on the frequency domain, the orders of integration were found to be around 1.60 for $U_{t}$ and $\log U_{t}$, and around 1.50 for $u_{t}$ and $u^{*}$, if the disturbances were white noise. Similar results were obtained when estimating through the time domain. Allowing weakly autocorrelated disturbances, either through autoregressions or through the Bloomfield (1973) exponential spectral model, the orders of integration were found to be higher, ranging in all cases between 1.70 and 2.20. 
Performing the tests of Robinson (1994) on these series, the results lead to the same conclusions, with the orders of integration ranging around 1.50 when modelling the disturbances as white noise, but obtaining higher values when allowing weak dependence on the disturbances. We also observed that in many cases more than one non-rejection case appeared. This is however not at all surprising, noting that when fractional hypotheses are entertained, some evidence supporting them may appear, because this might happen even when the unit root model is highly suitable. On the other hand, often the bulk of these hypotheses are rejected, suggesting that the optimal local power properties of the tests, shown by Robinson (1994), may be supported by reasonable performance against non-local alternatives.

The frequency domain approach used in this paper seems to be very unpopular amongst econometricians, and though there exist time domain versions of the Robinson's (1994) tests (cf, Robinson, 1991), the preference here for the frequency domain set-up is motivated by the somewhat greater elegance of formulae it affords when the Bloomfield model is used.

We should finally mention that we have not intended in this paper to investigate any model specification for the U.K. unemployment, rather to show that fractionally integrated models with Bloomfield (1973) exponential disturbances are feasible alternatives. The results indicate that all these series are nonstationary, with the orders of integration much higher than 1 . In fact, the above results show that $d$ is in practically all cases higher than 1.50 and thus, the standard approach of taking first differences still produces nonstationary series, which may then lead, when estimating by least squares, to erroneous conclusions.

Several other lines of research can be developed which should prove relevant to the analysis of these and other macroeconomic data. In particular, it would be worth to proceed to build up confidence intervals for the fractional differencing parameter, especially in the 
Bloomfield (1973) case. Also, the question of how best to extend this model to a multivariate set-up remains to be investigated. 


\section{REFERENCES}

Beran, J., 'Fitting long-memory models by generalized linear regression', Biometrika, 80, (1993), 817-822.

Bloomfield, P., 'An exponential model for the spectrum of a scalar time series', Biometrika, 60, (1973), 217-226.

Fox, R. and Taqqu, M.S., 'Large-sample properties of parameter estimates for strong dependent stationary Gaussian time series', Annals of Statistics, 14, (1986), 517-532.

Gil-Alana, L.A. and Robinson, P.M., 'Testing of unit root and other nonstationary hypotheses in macroeconomic time series', Journal of Econometrics, 80, (1997), 241-268.

Gil-Alana, L.A., 'Estimation of fractional ARIMA models for the U.K. unemployment', Centre for Economic Forecasting, London Business School, Discussion Paper 05-99, (1999a).

Gil-Alana, L.A., 'Testing the order of integration in the U.K. unemployment', Centre for Economic Forecasting, London Business School, Discussion Paper 06-99, (1999b).

Gil-Alana, L.A., 'Semi-parametric estimation of the fractional differencing parameter in the U.K. unemployment', Preprint. (1999c).

Granger, C.W.J., 'Long memory relationships and aggregation of dynamic models', Journal of Econometrics, 14, (1980), 227-238.

Robinson, P.M., 'Statistical inference for a random coefficient autoregressive model', Scandinavian Journal of Statistics, 5, (1978), 163-168.

Robinson, P.M., 'Testing for strong serial correlation and dynamic conditional heteroskedasticity in multiple regression', Journal of Econometrics, 47, (1991), 67-84.

Robinson, P.M., 'Efficient tests of nonstationary hypotheses', Journal of the American Statistical Association, 89, (1994), 1420-1437. 
Sowell, F., 'Maximum likelihood estimation of stationary univariate fractionally integrated time series models', Journal of Econometrics, 53, (1992), 165-188.

Velasco, C. and Robinson, P.M., 'Whittle Pseudo-Maximum likelihood estimation of nonstationary time series', Preprint. (1999). 


\begin{tabular}{|c|c|c|c|}
\hline \multicolumn{4}{|c|}{ TABLE $\mathbf{1}^{*}$} \\
\hline \multicolumn{4}{|c|}{ Estimation of the fractionally differencing parameter $\mathbf{d}$ for $\mathbf{U}_{\mathbf{t}}$} \\
\hline Fractionally integrated with $\mathrm{AR} \mathrm{u}_{\mathrm{t}}$ & \multicolumn{2}{|c|}{ Fractionally integrated with Bloomfield $\boldsymbol{u}_{\boldsymbol{t}}$} \\
\hline $\mathrm{AR}(\mathrm{p})$ & Value of $\mathrm{d}$ & Bloomfield $(\mathrm{m})$ & Value of $\mathrm{d}$ \\
\hline $\mathrm{p}=0$ & $\mathbf{1 . 6 6}$ & $\mathrm{m}=0$ & $\mathbf{1 . 6 5}$ \\
\hline $\mathrm{p}=1$ & $\mathbf{1 . 8 3}$ & $\mathrm{m}=1$ & $\mathbf{1 . 9 2}$ \\
\hline $\mathrm{p}=2$ & $\mathbf{1 . 9 2}$ & $\mathrm{m}=2$ & $\mathbf{2 . 0 9}$ \\
\hline $\mathrm{p}=3$ & $\mathbf{2 . 0 5}$ & $\mathrm{m}=3$ & $\mathbf{2 . 2 0}$ \\
\hline
\end{tabular}

*: $U_{t}$ corresponds to the number of people claiming unemployment benefits.

TABLE 2*

Estimation of the fractionally differencing parameter $d$ for $\log \mathbf{U}_{\mathbf{t}}$

\begin{tabular}{|c|c|c|c|}
\hline \multicolumn{2}{|c|}{ Fractionally integrated with $\mathrm{AR} \mathrm{u}_{\mathrm{t}}$} & \multicolumn{2}{|c|}{ Fractionally integrated with Bloomfield $\mathrm{u}_{\mathrm{t}}$} \\
\hline $\mathrm{AR}(\mathrm{p})$ & Value of $\mathrm{d}$ & Bloomfield $(\mathrm{m})$ & Value of $\mathrm{d}$ \\
\hline $\mathrm{p}=0$ & $\mathbf{1 . 6 3}$ & $\mathrm{m}=0$ & $\mathbf{1 . 6 2}$ \\
\hline $\mathrm{p}=1$ & $\mathbf{1 . 7 1}$ & $\mathrm{m}=1$ & $\mathbf{1 . 7 3}$ \\
\hline $\mathrm{p}=2$ & $\mathbf{1 . 7 4}$ & $\mathrm{m}=2$ & $\mathbf{1 . 8 0}$ \\
\hline $\mathrm{p}=3$ & $\mathbf{1 . 8 4}$ & $\mathrm{m}=3$ & $\mathbf{2 . 0 1}$ \\
\hline
\end{tabular}

*: $\log \mathrm{U}_{\mathrm{t}}$ is the $\log$ transformation of the number of people claiming unemployment benefits.

\section{TABLE 3*}

\section{Estimation of the fractionally differencing parameter $d$ for $u_{t}$}

Fractionally integrated with AR $u_{t}$

\begin{tabular}{|c|c|c|c|}
\hline \multicolumn{2}{|c|}{ Fractionally integrated with AR $u_{t}$} & \multicolumn{2}{|c|}{ Fractionally integrated with Bloomfield $u_{t}$} \\
\hline $\mathrm{AR}(\mathrm{p})$ & Value of $\mathrm{d}$ & Bloomfield $(\mathrm{m})$ & Value of $\mathrm{d}$ \\
\hline $\mathrm{p}=0$ & $\mathbf{1 . 5 0}$ & $\mathrm{m}=0$ & $\mathbf{1 . 4 9}$ \\
\hline $\mathrm{p}=1$ & $\mathbf{1 . 7 0}$ & $\mathrm{m}=1$ & $\mathbf{1 . 8 7}$ \\
\hline $\mathrm{p}=2$ & $\mathbf{1 . 8 8}$ & $\mathrm{m}=2$ & $\mathbf{2 . 0 8}$ \\
\hline $\mathrm{p}=3$ & $\mathbf{1 . 9 2}$ & $\mathrm{m}=3$ & $\mathbf{2 . 0 6}$ \\
\hline
\end{tabular}

*: $\mathrm{u}_{\mathrm{t}}$ is the number of people claiming unemployment benefits as a percentage of the workforce.

TABLE $4^{*}$

Estimation of the fractionally differencing parameter $d$ for $\mathbf{u}^{*}$

\begin{tabular}{|c|c|c|c|}
\hline \multicolumn{2}{|c|}{ Fractionally integrated with $\mathrm{AR} \mathrm{u}_{\mathrm{t}}$} & \multicolumn{2}{c|}{ Fractionally integrated with Bloomfield $\mathrm{u}_{\mathrm{t}}$} \\
\hline $\mathrm{AR}(\mathrm{p})$ & Value of $\mathrm{d}$ & Bloomfield $(\mathrm{m})$ & Value of $\mathrm{d}$ \\
\hline $\mathrm{p}=0$ & $\mathbf{1 . 4 9}$ & $\mathrm{m}=0$ & $\mathbf{1 . 4 9}$ \\
\hline $\mathrm{p}=1$ & $\mathbf{1 . 6 4}$ & $\mathrm{m}=1$ & $\mathbf{1 . 6 9}$ \\
\hline $\mathrm{p}=2$ & $\mathbf{1 . 7 1}$ & $\mathrm{m}=2$ & $\mathbf{1 . 8 3}$ \\
\hline $\mathrm{p}=3$ & $\mathbf{1 . 8 1}$ & $\mathrm{m}=3$ & $\mathbf{2 . 0 1}$ \\
\hline
\end{tabular}

$*: u_{t}^{*}$ is the logistic transformation of $u_{t}$. 


\section{TABLE $5^{*}$}

\begin{tabular}{|c|c|c|c|c|c|c|c|c|}
\hline \multicolumn{9}{|c|}{ Testing $d=d_{0}$ for $U_{t}$ with Bloomfield (1973) exponential spectral disturbances } \\
\hline \multirow[b]{2}{*}{$\mathbf{m}$} & \multirow[b]{2}{*}{$\mathbf{z}_{\mathbf{t}}$} & \multicolumn{7}{|c|}{ Values of $d_{0}$} \\
\hline & & 1.00 & 1.20 & 1.40 & 1.50 & 1.60 & 1.80 & 2.00 \\
\hline \multirow{3}{*}{$\mathbf{m}=\mathbf{0}$} & No regressors & 18.75 & 6.26 & -1.30 & -3.43 & -4.86 & -6.50 & -7.36 \\
\hline & An intercept & 34.89 & 24.18 & 11.36 & 5.87 & 1.60 & -3.62 & -6.06 \\
\hline & A time trend & 34.88 & 24.15 & 11.27 & 5.78 & 1.51 & -3.68 & -6.10 \\
\hline \multirow{3}{*}{$\mathbf{m}=1$} & No regressors & 11.54 & 6.44 & 2.12 & 0.38 & -0.85 & -3.05 & -4.29 \\
\hline & An intercept & 18.24 & 13.42 & 9.31 & 6.97 & 4.91 & 1.24 & -1.27 \\
\hline & A time trend & 18.24 & 13.36 & 9.01 & 6.81 & 4.74 & 1.36 & -1.21 \\
\hline \multirow{3}{*}{$m=2$} & No regressors & 7.63 & 5.16 & 2.76 & 1.35 & 1.02 & -0.68 & -2.34 \\
\hline & An intercept & 11.22 & 8.10 & 5.63 & 5.10 & 3.18 & 2.05 & -0.01 \\
\hline & A time trend & 11.23 & 8.04 & 5.50 & 4.92 & 3.82 & 1.83 & 0.46 \\
\hline \multirow{3}{*}{$\mathbf{m}=\mathbf{3}$} & No regressors & 5.42 & 5.38 & 3.54 & 1.52 & 1.38 & 1.16 & 0.54 \\
\hline & An intercept & 8.96 & 7.24 & 6.54 & 5.10 & 4.30 & 2.95 & 0.31 \\
\hline & A time trend & 8.94 & 7.21 & 6.46 & 5.01 & 4.20 & 2.95 & 0.41 \\
\hline
\end{tabular}

*: $U_{t}$ is the number of people claiming unemployment benefits. In bold: The non-rejection values at the $95 \%$ significance level.

\section{TABLE 6"}

Testing $d=d_{0}$ for $\log U_{t}$ with Bloomfield (1973) exponential spectral disturbances

\begin{tabular}{|c|c|c|c|c|c|c|c|c|}
\hline \multicolumn{2}{|c|}{} & \multicolumn{7}{|c|}{ Values of $\mathbf{d}_{\mathbf{0}}$} \\
\hline $\mathbf{m}$ & $\mathbf{z}_{\mathbf{t}}$ & $\mathbf{1 . 0 0}$ & $\mathbf{1 . 2 0}$ & $\mathbf{1 . 4 0}$ & $\mathbf{1 . 5 0}$ & $\mathbf{1 . 6 0}$ & $\mathbf{1 . 8 0}$ & $\mathbf{2 . 0 0}$ \\
\hline \hline \multirow{3}{*}{$\mathbf{m}=\mathbf{0}$} & No regressors & $\mathbf{- 0 . 0 8}$ & -3.39 & -5.23 & -5.85 & -6.34 & -7.08 & -7.60 \\
\cline { 2 - 9 } & An intercept & 28.06 & 17.49 & 7.38 & 3.47 & $\mathbf{0 . 4 4}$ & -3.43 & -5.48 \\
\cline { 2 - 9 } & A time trend & 28.07 & 17.40 & 7.21 & 3.27 & $\mathbf{0 . 2 5}$ & -3.59 & -5.61 \\
\hline \hline \multirow{3}{*}{$\mathbf{m}=\mathbf{1}$} & No regressors & $\mathbf{- 0 . 1 4}$ & -2.27 & -3.61 & -4.02 & -4.31 & -4.94 & -5.45 \\
\cline { 2 - 9 } & An intercept & 13.09 & 8.30 & 4.60 & 2.89 & $\mathbf{1 . 3 2}$ & $\mathbf{- 1 . 1 7}$ & -3.04 \\
\cline { 2 - 9 } & A time trend & 13.12 & 8.62 & 4.33 & 2.57 & $\mathbf{1 . 2 8}$ & $\mathbf{- 1 . 2 9}$ & -3.09 \\
\hline \hline \multirow{3}{*}{$\mathbf{m}=\mathbf{2}$} & No regressors & $\mathbf{- 0 . 1 9}$ & $\mathbf{- 1 . 2 4}$ & -3.33 & -3.61 & -3.91 & -3.97 & -4.50 \\
\cline { 2 - 9 } & An intercept & 7.22 & 3.48 & 2.75 & $\mathbf{1 . 2 2}$ & $\mathbf{0 . 0 5}$ & $\mathbf{- 1 . 4 9}$ & -2.29 \\
\cline { 2 - 9 } & A time trend & 7.25 & 3.34 & 2.44 & $\mathbf{0 . 8 4}$ & $\mathbf{1 . 4 3}$ & $\mathbf{- 0 . 3 4}$ & $\mathbf{- 0 . 8 1}$ \\
\hline \multirow{3}{*}{$\mathbf{m}=\mathbf{3}$} & No regressors & $\mathbf{0 . 2 1}$ & $\mathbf{- 0 . 2 4}$ & $\mathbf{- 0 . 4 8}$ & -2.17 & -3.04 & -3.94 & -4.83 \\
\cline { 2 - 9 } & An intercept & 8.84 & 7.55 & 2.55 & $\mathbf{1 . 7 4}$ & $\mathbf{1 . 5 0}$ & $\mathbf{0 . 9 0}$ & $\mathbf{- 0 . 8 6}$ \\
\cline { 2 - 8 } & A time trend & 8.84 & 7.45 & 2.32 & $\mathbf{1 . 4 5}$ & $\mathbf{1 . 2 1}$ & $\mathbf{0 . 7 8}$ & $\mathbf{- 0 . 9 5}$ \\
\hline
\end{tabular}

*: $\log U_{t}$ is the $\log$ transformation of the number of people claiming unemployment benefits. In bold: The nonrejection values at the $95 \%$ significance level. 


\section{TABLE 7 *}

\begin{tabular}{|c|c|c|c|c|c|c|c|c|}
\hline \multicolumn{9}{|c|}{ Testing $d=d_{0}$ for $u_{t}$ with Bloomfield (1973) exponential spectral disturbances } \\
\hline \multirow[b]{2}{*}{$\mathbf{m}$} & \multirow[b]{2}{*}{$\mathbf{z}_{\mathrm{t}}$} & \multicolumn{7}{|c|}{ Values of $d_{0}$} \\
\hline & & 1.00 & 1.20 & 1.40 & $\mathbf{1 . 5 0}$ & 1.60 & $\mathbf{1 . 8 0}$ & 2.00 \\
\hline \multirow{3}{*}{$\mathbf{m}=\mathbf{0}$} & No regressors & 16.21 & 4.42 & -2.18 & -4.00 & -5.23 & -6.66 & -7.43 \\
\hline & An intercept & 29.76 & 15.89 & 3.63 & -0.39 & -3.14 & -6.13 & -7.43 \\
\hline & A time trend & 29.76 & 15.85 & 3.55 & -0.48 & -3.22 & -6.19 & -7.48 \\
\hline \multirow{3}{*}{$\mathbf{m}=1$} & No regressors & 10.34 & 5.55 & 1.35 & -0.15 & -1.44 & -3.31 & -4.51 \\
\hline & An intercept & 16.81 & 12.54 & 7.89 & 5.88 & 3.65 & 0.69 & -1.70 \\
\hline & A time trend & 16.82 & 12.49 & 7.76 & 5.70 & 3.79 & 0.73 & -1.78 \\
\hline \multirow{3}{*}{$m=2$} & No regressors & 6.74 & 4.39 & 2.21 & 0.78 & -0.25 & -1.53 & -2.10 \\
\hline & An intercept & 12.45 & 8.81 & 6.53 & 4.02 & 3.46 & 2.48 & 0.69 \\
\hline & A time trend & 12.46 & 8.75 & 6.38 & 3.80 & 3.20 & 2.15 & 0.24 \\
\hline \multirow{3}{*}{$\mathbf{m}=\mathbf{3}$} & No regressors & 4.81 & 2.14 & 2.06 & 0.97 & -0.45 & -2.14 & -2.79 \\
\hline & An intercept & 7.31 & 6.40 & 3.13 & 2.20 & 1.41 & 0.30 & -0.22 \\
\hline & A time trend & 7.31 & 6.37 & 3.05 & 2.10 & 1.32 & 0.34 & -2.12 \\
\hline
\end{tabular}

*: $\mathrm{u}_{\mathrm{t}}$ is the number of people claiming unemployment benefits as a percentage of the workforce. In bold: The non-rejection values at the $95 \%$ significance level.

\section{TABLE 8*}

Testing $d=d_{0}$ for $u^{*}{ }_{t}$ with Bloomfield (1973) exponential spectral disturbances

\begin{tabular}{|c|c|c|c|c|c|c|c|c|}
\hline \multicolumn{2}{|c|}{} & \multicolumn{7}{|c|}{ Values of $\mathbf{d}_{\mathbf{0}}$} \\
\hline $\mathbf{m}$ & $\mathbf{z}_{\mathbf{t}}$ & $\mathbf{1 . 0 0}$ & $\mathbf{1 . 2 0}$ & $\mathbf{1 . 4 0}$ & $\mathbf{1 . 5 0}$ & $\mathbf{1 . 6 0}$ & $\mathbf{1 . 8 0}$ & $\mathbf{2 . 0 0}$ \\
\hline \hline \multirow{3}{*}{$\mathbf{m}=\mathbf{0}$} & No regressors & $\mathbf{- 0 . 0 7}$ & -3.49 & -5.30 & -5.91 & -6.39 & -7.11 & -7.62 \\
\cline { 2 - 9 } & An intercept & 24.59 & 12.56 & 2.90 & $\mathbf{- 0 . 3 2}$ & -2.63 & -5.37 & -6.74 \\
\cline { 2 - 9 } & A time trend & 24.60 & 12.48 & 2.74 & $\mathbf{- 0 . 5 0}$ & -2.80 & -5.52 & -6.88 \\
\hline \multirow{3}{*}{$\mathbf{m}=\mathbf{1}$} & No regressors & $\mathbf{- 0 . 1 2}$ & -2.26 & -3.64 & -4.03 & -4.45 & -5.03 & -5.37 \\
\cline { 2 - 9 } & An intercept & 12.61 & 8.32 & 4.19 & 2.50 & $\mathbf{0 . 9 6}$ & $\mathbf{- 1 . 4 5}$ & -3.22 \\
\cline { 2 - 9 } & A time trend & 12.64 & 8.20 & 4.28 & 2.49 & $\mathbf{1 . 1 8}$ & $\mathbf{- 1 . 4 0}$ & -3.27 \\
\hline \hline \multirow{3}{*}{$\mathbf{m}=\mathbf{2}$} & No regressors & $\mathbf{- 0 . 1 6}$ & $\mathbf{- 1 . 5 5}$ & -3.19 & -3.52 & -3.56 & -4.12 & -4.65 \\
\cline { 2 - 9 } & An intercept & 6.69 & 4.65 & 3.84 & 2.18 & $\mathbf{0 . 9 1}$ & $\mathbf{- 0 . 7 6}$ & $\mathbf{- 1 . 6 3}$ \\
\cline { 2 - 9 } & A time trend & 6.72 & 4.52 & 3.51 & 2.77 & 2.35 & $\mathbf{0 . 3 9}$ & $\mathbf{- 0 . 9 6}$ \\
\hline \hline \multirow{3}{*}{$\mathbf{m}=\mathbf{3}$} & No regressors & $\mathbf{- 0 . 2 9}$ & $\mathbf{- 0 . 6 6}$ & $-\mathbf{1 . 0 6}$ & $\mathbf{- 1 . 6 9}$ & -3.92 & -4.31 & -5.13 \\
\cline { 2 - 8 } & An intercept & 5.62 & 4.10 & 2.34 & $\mathbf{1 . 4 2}$ & $\mathbf{1 . 2 3}$ & $\mathbf{- 1 . 1 2}$ & $\mathbf{- 1 . 2 2}$ \\
\cline { 2 - 8 } & A time trend & 5.63 & 4.02 & 2.12 & $\mathbf{1 . 1 7}$ & $\mathbf{1 . 0 4}$ & $\mathbf{- 1 . 0 1}$ & $\mathbf{- 1 . 7 9}$ \\
\hline
\end{tabular}

$: \mathrm{u}_{\mathrm{t}}^{*}$ is the logistic transformation of $\mathrm{u}_{\mathrm{t}}$. In bold: The non-rejection values at the $95 \%$ significance level. 
